Article

\title{
An Artificial Intelligence Empowered Cyber Physical Ecosystem for Energy Efficiency and Occupation Health and Safety
}

\author{
Petros Koutroumpinas ${ }^{1}$, Yu Zhang ${ }^{2, *}$, Steve Wallis ${ }^{3}$ and Elizabeth Chang ${ }^{2}$ \\ 1 Faculty of Engineering, Monash University, Melbourne, VIC 3800, Australia; pkou0003@student.monash.edu \\ 2 School of Business, University of New South Wales, Canberra, ACT 2612, Australia; e.chang@adfa.edu.au \\ 3 Fleetwood Corporation Limited, Perth, WA 6004, Australia; stevew@glyde.net.au \\ * Correspondence: m.yuzhang@unsw.edu.au
}

check for

updates

Citation: Koutroumpinas, P.; Zhang, Y.; Wallis, S.; Chang, E. An Artificial Intelligence Empowered Cyber Physical Ecosystem for Energy Efficiency and Occupation Health and Safety. Energies 2021, 14, 4214. https://doi.org/10.3390/en14144214

Received: 17 April 2021

Accepted: 8 July 2021

Published: 12 July 2021

Publisher's Note: MDPI stays neutral with regard to jurisdictional claims in published maps and institutional affiliations.

Copyright: (C) 2021 by the authors. Licensee MDPI, Basel, Switzerland. This article is an open access article distributed under the terms and conditions of the Creative Commons Attribution (CC BY) license (https:/ / creativecommons.org/licenses/by/ $4.0 /)$.

\begin{abstract}
Reducing energy waste is one of the primary concerns facing Remote Industrial Plants (RIP) and, in particular, the accommodations and operational plants located in remote areas. With the COVID-19 pandemic continuing to attack the health of workforce, managing the balance between energy efficiency and Occupation Health and Safety (OHS) in the workplace becomes another great challenge for the RIP. Maintaining this balance is difficult mainly because a full awareness of the OHS will generally consume more energy while reducing the energy cost may lead to a less effective OHS, and the existing literature has not seen a system that is designed for the RIPs to conserve energy usage and improve workforce OHS simultaneously. To bridge this gap, in this paper, we propose an AI Empowered Cyber Physical Ecosystem (AECPE) solution for the RIPs, which integrates Cyber-Physical Systems (CPS), artificial intelligence, and mobile networks. The preliminary results of lab experiments and field tests proved that the AECPE was able to help industries reduce the corporate annual energy cost that is worth millions of dollars, optimise the environmental conditions, and improve OHS for all workers and stakeholders. The implementation of the AECPE can result in efficient energy usage, reduced wastage and emissions, environment-friendly operations, and improved social reputation of the industries.
\end{abstract}

Keywords: cyber-physical system; ecosystem; remote industries; OHS; energy efficiency; smart meter; artificial intelligence; COVID-19

\section{Introduction}

Maintaining a delicate balance between energy efficiency and Occupation Health and Safety (OHS) [1] has been a challenge facing large enterprises and particularly the Remote Industrial Plants (RIP), such as mining campsites and other resources industries and remote industrial facilities in regional and countryside areas. With extreme weather conditions around the world, energy powered air conditioning systems need to be properly managed in order to prevent uninhabitable situations from happening.

As examples: when the temperature outside of a mining campsite is around $40{ }^{\circ} \mathrm{C}$, it could exceed $50^{\circ} \mathrm{C}$ indoors; one person working after hours turning on the entire site's or building's power and lights instead of the one specific spot that is needed; and malfunctioning air-conditioning units in an operational plant could lead all staff to experience stress due to high or low temperatures. The extreme weather conditions require a constant supply of energy with balanced heating or cooling to ensure that OHS standards are met for the sake of all employees and visitors as well as stakeholders.

In addition, the challenge extends to the issues of appropriate lighting, ventilation, air-quality, and humidity control. Regardless of whether the majority of RIPs are reliant on fossil fuels to generate power and are off-site, off-grid with no electrical networks, or in the city where the lights are on in continuous and constant mode. With massive energy consumption, the difficulty lies in how to properly manage the energy cost whilst ensuring OHS conditions are optimal for the workforce. 
In order to address the above issues and risks in the RIPs, this paper presents an AI Empowered Cyber Physical Ecosystem (AECPE), which is a cost-effective, smart digital ecosystem solution that integrates Cyber-Physical Systems (CPS), mobile networks, and artificial intelligence. The AECPE is designed to intelligently provide personalised services, including a live-able temperature, refrigerating perishables, water temperature control, electrical appliances, lighting, ventilation, and room humidity, by detecting the occupancy of rooms or facilities and monitoring the habitable environments.

This differs from any existing CPS or Internet of Things (IoT) since its focus is on making the computing elements of the system coordinate more closely with the sensors and the actuators to achieve a higher level of system intelligence and efficiency in monitoring and controlling the cyber and physical environments. In addition, the mobile networks and AI enable the system and devices to exchange information, analyse data and produce realtime energy usage and environmental awareness. The AECPE leverages communications among sensors, smart meters, and AI modules through mobile networks to create a cyber physical ecosystem that not only reduces electricity wastage and carbon emissions but also fulfils OHS compliance at the same time.

The contribution of the proposed AECPE lies more towards its practical applications than theoretical level, which is summarised from three aspects. Firstly, more OHS profile entries are considered in the AECPE since the system is designed for remote area industrial plants and accommodations, including indoor/outdoor and historical/forecast temperature, lighting, air quality, ventilation, humidity, personal use preferences, and movement from area to area. Secondly, the actuators are managed by machine-learning based algorithms that require minimal human intervention, and that take into account valuable real data collected from test sites, such as user preferences, room occupancy and personal movement which can be used for not only maintaining the balance between energy efficiency and OHS but also contact tracing to offer proactive responses during the COVID-19 pandemic. Finally, the ACEPE has been tested on real data in a real-life environment with highly demanding accommodation and a physically stressful outdoor environment, and the results have shown success in reducing the electricity consumption while maintaining a user-friendly living environment.

The remainder of this paper is structured as follows. Section 2 presents the practical issues facing the current industries and technologies. Section 3 introduces the framework of the proposed AECPE. Section 4 describes the design of the AECPE in detail, followed by the prototype setup and field testing of the system in Section 5. Lastly, Section 6 presents the conclusions and directions for future study.

\section{Related Work}

\subsection{Cyber-Physical Systems}

A Cyber Physical System (CPS) is a system that provides new ways for humans to interact with the cyber and the physical world [2]. A CPS generally contains two main parts. The first is real-time connectivity to the physical world allowing continual information feedback, and the second is intelligent data management and analytics. Instead of using a two-part structure, a five-part structure of CPS was proposed [3]. They are (i) smart connection, (ii) data-to-information conversion, (iii) a cyber level, (iv) cognition level, and finally (v) configuration level. Each of these five sections will have subsections.

A smart connection should involve sensors and metering wireless networks and plug and play connectivity. This section does not have to be online if not necessary. A Zigbee protocol can be used for this section. Data analytics and desegregation will take part in the data-to-information section. There are two choices of how to go about this. The analytics can occur on-site where the data was collected or can be send to the cloud for the analytics to occur there. On-site analytics require for there to be a server framework created on said site. On the other hand, for the data to be send to the cloud could put a strain on devices if they are running on a battery or could put excess strain on the bandwidth available. 
In a case study, it was seen that the power draw of the CPS system could be reduced by up to $70 \%$ if the collected data were first compressed before being sent to the cloud [4]. This promotes the idea that some amount of on-site pre-processing will have to occur. The cyber level will allow for further analysis to occur and models to be created to be able to identify similarities or variations within the incoming data. Next, the cognition level will allow the incoming data to be visualized for the user. Monitoring will play an important role in the future of CPS as it will enable real-time alerts and corrective actions depending on what is needed by the system [5].

The final level is the configuration level, which includes a large amount of machine learning algorithms so that the system can be self-adjustable, self-optimizing, and selfconfiguring. If the system can achieve these requirements, it will be able quickly adjust as the system grows and become more complex throughout its operation. These five steps can act as a good road map in the future implementation of new Cyber Physical Systems [3] .

In terms of CPS applications, these have been used in many sectors, such as aviation, IT, transport, and medical fields. The medical field has seen multiple case studies. One such study proposed the Medical Cyber Physical System or MCPS [6]. The main problems that a system like this would face are reliability, security, and safety requirements, which require further human interactions to obtain the best use out of the system. The paper also draws attention to the impact that cyber attacks can have as well as the need for a combined design methodology to resolve the design challenges of long-term learning and self-adaption of MCPS.

A further case study into the applications of CPS in the medical field was applied in a house environment [7] to improve the quality of life for elderly patients or other patients. What they proposed was the application of a camera and sensor network for real time surveillance of the patient to allow better and more efficient care. This would also allow real-time scheduling and management of resources-for example, the dispensing of medication. A point that this paper raised is aligned with another CPS research, which claimed that any lapses in the security and communication of the MCPS could put lives at risk [6]. The MCPS was also used to control an Analgesic Infusion pump [8].

Their application used a spatio-temporal partial differential equation and a PKA model to determine the concentration of a medication in a patient's blood stream. If the concentration drops too low, then the pump will be told to increase the output of medication and vice versa if the concentration is too high. This way, if there is a high enough confidence in the system, the human error can be removed, and the response time to the changes in concentration will be detected quicker by the software than by the human user, thus, improving the safety for the patient.

While CPS has been used as the framework to connect the cyber and physical worlds, many system control approaches have been proposed. An artificial bee colony optimisation algorithm was proposed to simultaneously maximise comfort whilst minimising energy usage [9]. Fuzzy controllers were used in the system where the input for the controllers was the difference between the input user parameters and the actual parameters, and the output is the required power to affect the conditions. This solution aims to address the Comfort index, which applies on the suitability of environmental conditions for physical activity. Applying fuzzy logic for system control has become more common as the need for energy saving is rising.

A fan coil unit was proposed as a way of transporting heat from the air into a coolant fluid [10]. In their experiment, the controlled variables were the room temperature and the room humidity. It was shown that fuzzy controls successfully managed the previous variables, whereas the Proportional Integral Derivative control failed to do so, which uses more energy. It can also be used for multiple system control, such as managing the room temperature control whilst taking into account the dew point [11]. This study showed that fuzzy logic was an easy way of solving this problem without getting too involved in the physical variables. Fuzzy logic has been shown to be an effective solution when dealing with a temperature control problem in certain situations. 
However, fuzzy logic lacks the ability to make future predictions based on historical information, such as how much energy will be used in the future leading to future costs being known. Machine learning, on the other hand, excels at this given that a large amount of data is collected and ready for use [12]. In our project, three years of room temperature data was collected and provided for experiments, and the outdoor daily temperature for the region can also be accessed online [13], thus, making the machine learning a more effective solution than fuzzy logic for this situation.

\subsection{Cyber Security}

In any situation where a wireless connection is involved, cyber security is an issue that has to be taken into account. Different types of attack can occur. A denial-of-service (DoS) attack involves rendering a service, a computer, or other devices unavailable to the intended users by overwhelming a targeted machine or service with requests until normal requests cannot come through [14]. Sites, such as shopping centres, airports, universities, and other public locations, are vulnerable to these types of attacks due to their broadcasting nature [15].

These attacks can also slow down systems and make them use stale data [16]. In this case, it would drive up the energy usage as the optimisation algorithm relies on having the most current data. There is also a risk of the room not being at the correct temperature when the worker arrives, which would be an OHS risk. A CPS could also face replay attacks, which involve an intercepted message being delayed or intercepted to misdirect the receiver [17]. This attack could occur through the end sensors of the CPS, where the collected data could be collected and then duplicated [18].

A possible way to combat this attack is to use a random authentication signal in the control system [18] or encryption keys [17]. Another attack is a false data injection attack, which can happen in a power grid [19]. This attack also involves an attack on the sensors; however, in this case, it involves compromising the sensor reading in such a way that undetected errors are introduced into the calculations of state variables and values [20].

\subsection{Occupational Health and Safety}

Occupational Health and Safety (OHS) is the study of workplace influences on health and well-being, including identifying the environment and mechanism causes of imbalances related to human biological, chemical, and physical pollutants in the workplace and providing a customised and occupational health and safety compliant environment for workers, including remote operations. The Occupational Safety and Health Act and OHS risk management supported by the Occupational Safety and Health Regulations in each country are used to ensure that habitable conditions are met for the workers.

Occupational health is as important as physical health, and we developed mechanisms for better occupational health using data captured through CPS devices to infer and identify risk factors (i.e., stress, fatigue, and mental illness). There have been some studies, applications, and devices focusing on CPS (listed as follows); however, they all showed their limitations in considering the OHS.

- $\quad$ Smart metering from EKM Metering (US) and Meazon (EU) are great at monitoring energy and water usage to extremely high accuracy but they do not offer services to improve OHS [21].

- Paxton (UK) offers wireless door handles for access control but does not consider OHS [22].

- $\quad \mathrm{KBE}$, one of the world's largest manufacturers for energy saving buildings, focuses only on materials used for housing but not OHS [23].

- USA, China, and EU have worked on energy optimisation through the IoT coupled with renewable energy for remote and rural communities to improve lifestyle through energy supply, but do not focus on the corporate social responsibility for OHS of their workers or tenants [24,25]. 
- Smart Cities and smart homes are other areas close to this proposed project. An application of Smart Cities [26] to remote areas investigated improve lifestyle through energy efficient appliances but not OHS in the corporate environment.

\section{AI Empowered Cyber Physical Ecosystem (AECPE) Solution Architecture}

The AECPE solution architecture is proposed based on four main sections, namely OHS Profile data acquisition, ML-based optimiser, a smart metering system, and cyber security and COVID-19 response, to be able to fulfil the needs of energy efficiency, OHS requirements, and COVID-19 responses. The overall architecture is demonstrated in Figure 1 in which the sections are framed by dashed lines in different colours.

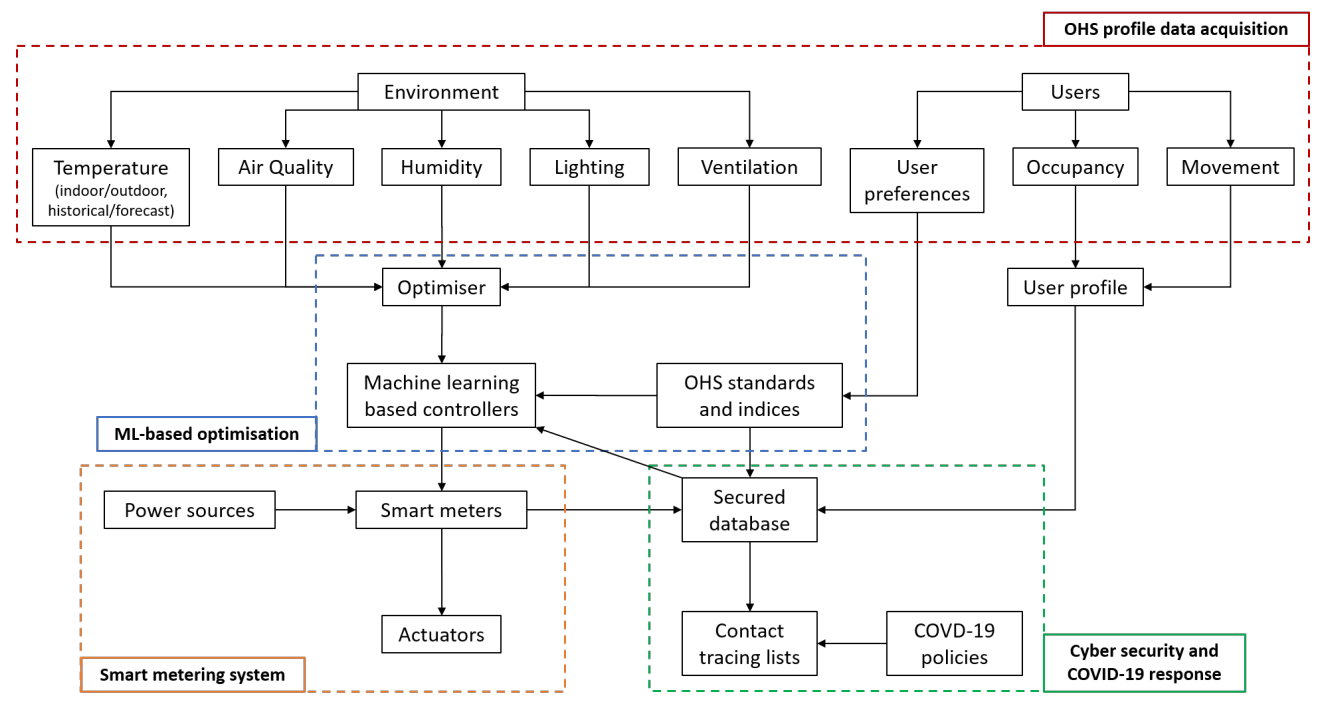

Figure 1. The AI Empowered Cyber Physical Ecosystem (AECPE) solution architecture.

Specifically, the environment data and user information that concern the OHS requirements are collected as an OHS profile input in the OHS profile data acquisition section. The environment profile is entered to an optimiser, and the user preferences together with the OHS standards and indices, are fed to the machine-learning-based controllers for a series of analysis, optimisation, and prediction in the ML-based optimiser. These results are input to the smart metering system in which the smart meters request the required electricity from the power sources and, meanwhile enable the actuators to switch the devices on and off (air conditions, lighting, ventilation, etc.).

All the data mentioned above is stored in a secured database, and this database can also talk to the ML-based controllers for continuous learning. On the other hand, the room occupancy and user movement are also monitored to create a user profile, which is also stored in the database for COVID-19 responses in terms of contact tracing according to COVID-19 policies. The details of these sections will be described later.

\section{AECPE Design}

\subsection{OHS Profile Data Acquisition}

The OHS profile is collected through monitoring the site environment and users in real time for correct decision making, for both human decisions and machine decisions. To this end, the system will need to be able to access any stored data or any processed data. Middle-ware will also need to be developed and applied over the smart gateway system to help with management of the network and to make any monitoring applications easier to run. Monitoring should also be able to allow for the use of regression algorithms combined with time series algorithms that can predict future energy usage using the past data. This will allow for the better distribution of resources leading to reductions in operations costs. Information to help with COVID-19 prevention can also be collected as part of the OHS Profile. 
The OHS profile includes indoor and outdoor temperature, as well as temperature in the past and climate forecast for the near future, air quality, humidity, lighting, ventilation, personal user preferences, room occupancy, and user movement.

Having a room at a comfortable temperature is important, as heat stroke can occur easily in an environment that is under a near constant heat wave. A heat wave is defined as an area whose temperature over $32.2^{\circ} \mathrm{C}$ for 3 days or more in a row, which is a criteria that is met by the chosen site for multiple months every year [27]. Having this heat wave period greatly increases the risk of heat stroke occurring. Heat stroke is when the body's core temperature goes above $40{ }^{\circ} \mathrm{C}$ [27]. The risk of this occurring is even higher when workers return after a $10-\mathrm{h}$ shift of physical labour. Where the temperature of the room is kept exactly will be subject to the user when they are in the room. This is done through either the remote control of the air-conditioners for a one time change or by setting a new permanent temperature through the app.

Another aspect that needs to be accounted for is the dew point and humidity of the area, which changes greatly over time as there are large swings in the daily temperature and the relative humidity. The dew-point must be precisely controlled. This is due to the fact that it has been observed (on site) that, if the temperature in the room is left beneath the dew point, and moisture starts to build and mould can occur. If mould builds in a room and it is not cleaned fast enough, it can cause permanent structural damage.

It can cost up to 2000 dollars to have mould cleaned, and it can render a block of four apartments unlivable for up to 2 weeks. Therefore, whilst taking into account that the temperature has to be kept cool enough to be lived in, the room also has to be allowed to be heated above the dew point temperature long enough for moisture to evaporate to stop mould from forming. Controlling humidity will also help to ensure that the air quality in the rooms is maintained at a good level.

The capture kit containing the sensors and actuators that allow OHS to be monitored was installed in the accommodation to start collecting data. This captured data plays a crucial role in delivering the balance between energy efficiency and OHS outcomes. A secure communication between the sensors, the kit, and the cloud servers was established with security mechanisms to maintain data security and to ensure the users' privacy.

Sample temperature data from the whole site was collected by both indoor and outdoor thermometers, including individual room thermometers and inbuilt air-conditioning thermometers as well as checked against online meteorological data for the region. Both indoor and outdoor temperatures are required, as there is a tendency for the internal temperature to be higher than external temperatures. At higher temperatures, there is a strong correlation between the two temperatures [28] that is not there at lower temperatures.

As the site is located in a region that has average maximum temperatures reaching above $30{ }^{\circ} \mathrm{C}$ for six months of the year and mid to high $20^{\circ} \mathrm{C}$ for the other half year [29], this linear correlation occurs from when the temperature is greater than $12.7^{\circ} \mathrm{C}$, which is well below even the average minimum temperature for the site. Collection and analysis of this data will lead to a better understanding of localised energy usage and OHS needs. The third stage will provide the roster and demographic data. This step also includes pre-process data that includes data cleansing, data transformation, and labelling process. After pre-processing, data are fed into the AI and machine learning algorithms for analysis.

The overall data collection and analysis are demonstrated in Figure 2. This flowchart shows how incoming data from energy usage and sensor data is collected and processed, to generate reports and generate new commands to be able to run a more efficient site. 


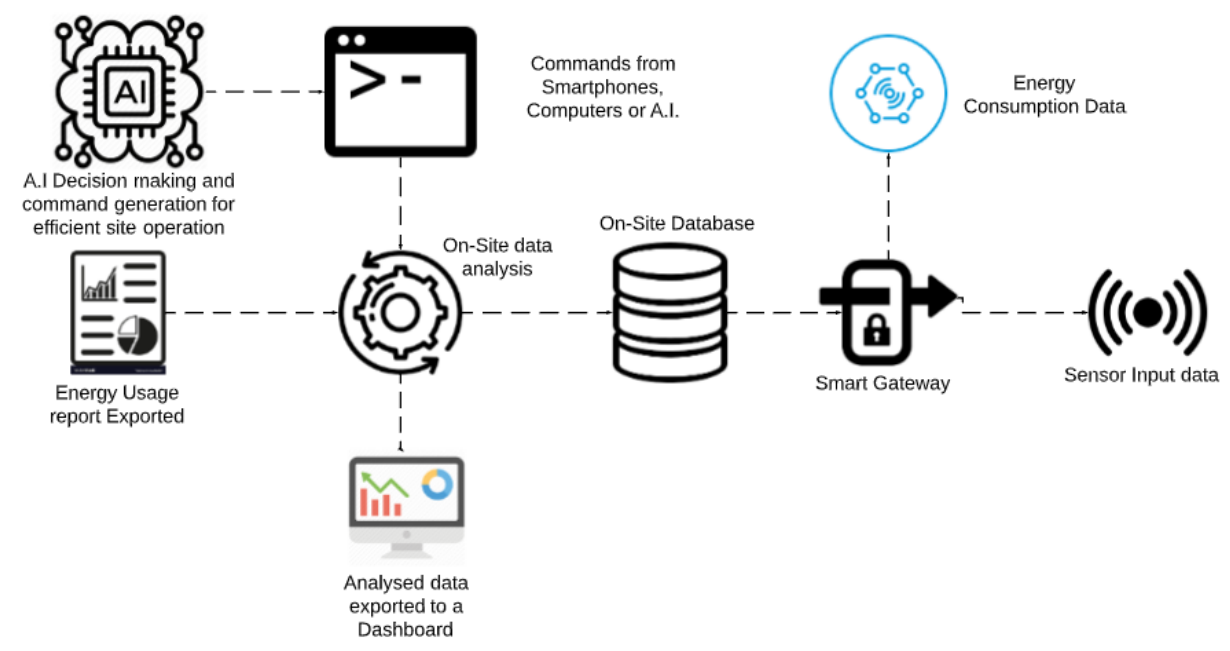

Figure 2. Data collection and analysis.

\subsection{Smart Metering System}

Integrating smart meters within a mesh network will allow for a flexible system that can be monitored and controlled in real-time to meet any needs that a site might require. Monitoring the energy consumption and system performance will allow for precise device scheduling to manage energy consumption while retaining health and well-being conditions at its most optimal. This will be done by analysing rosters for people coming into the plant or accommodation sites. From those rosters, the arrival and departure dates of the workers will be extracted along with the shifts that they will be working, through which the indoor temperature will be managed to be as efficient as possible.

This will be done by taking environmental data into account, such as the external temperature and humidity; as well as room data, such as the internal temperature and time until the return of the worker to the room. Energy consumption monitoring and scheduling techniques aim to reduce overall energy consumption without compromising the level of comfort. Two-way communication allows operation control over appliances, hence, enabling the operation of energy consumption scheduling. The techniques incorporate the aggregated demand to optimise electricity consumption across the plant and accommodation sites. The design of the AECPE physical layout is demonstrated in Figure 3.

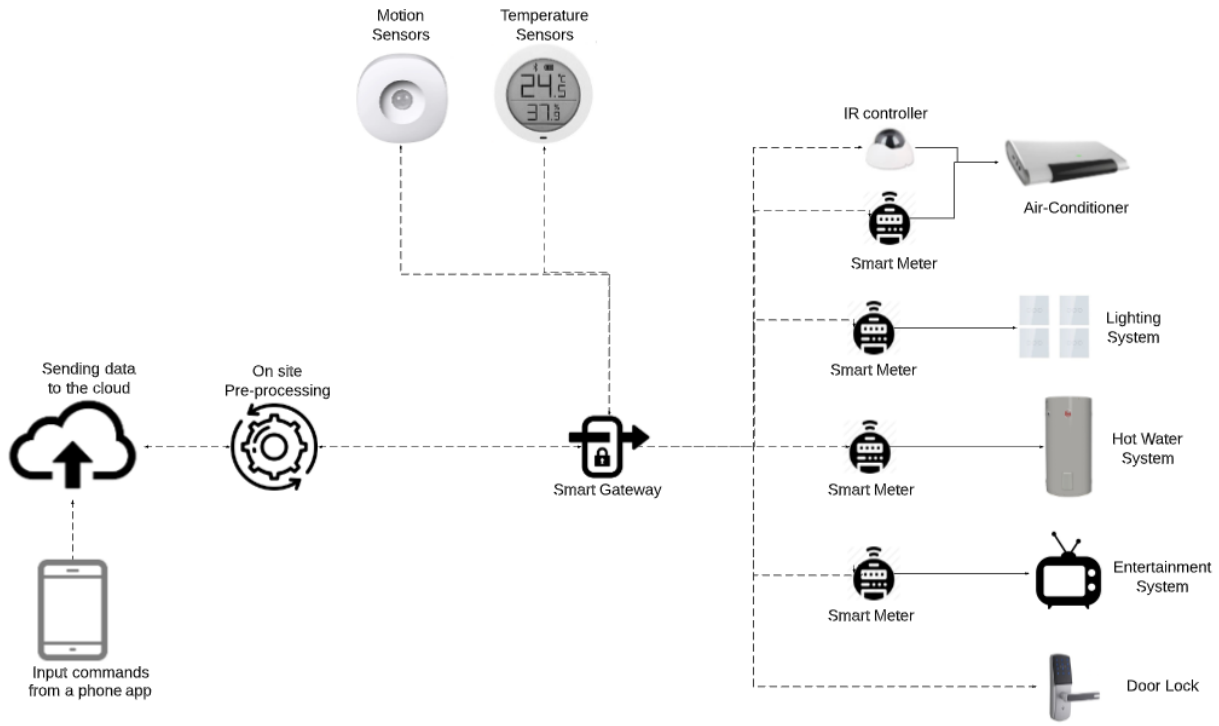

Figure 3. The on-site layout of devices and smart meters. 
In this figure, the dotted lines represent wireless connections, whether they are Zigbee connections between the gateway and the meters and sensors or Ethernet connections between the gateway and pre-processing. The pre-processing that will occur is to combine the different data sets of energy usage and weather conditions so that the regression algorithm can act on them. The solid lines represent actual wiring connections where electricity is fed to each of the machines or systems. Zigbee is a good choice for the device control section of the system as it is a low energy use protocol, which means that a device can run on a battery for a long time. Zigbee can also connect to up to 4160 plus devices [30], which means that the mesh network can be stretched to cover any size site that may be required.

\subsection{ML-Based Optimisation}

Machine learning techniques were used to analyse the collected sensor data and OHS profiles to maintain the energy efficiency and workplace environment. Linear Regression models allow cause-effect relationships to be identified using past energy usage data combined with daily temperature information collected online. This will be done so that it is known at what temperature setting the air conditioning units need to be running for the most efficient energy usage based on the outdoor temperatures and required indoor temperature. This will result in energy saving while maintaining the health and well being for occupants.

Time-series forecasting algorithms will also be employed to enable energy consumption scheduling (the next milestone) and adaptive actions for health and well-being (mainly keeping the temperature to a comfortable level). Time-series forecasting will also allow an estimation of future energy consumption and future energy costs for informed decision making. The formation of mould is another risk that needs to be mitigated. Mould forms when the temperature of the room is kept below the dew point for a consistent period. If this happens, the kit will adjust to increase the temperature to stop mould formation-provided it does not go against any OHS requirements.

A regression algorithm predicts the output values based on the input features from the data fed into the system [31]. Specifically, a multivariate regression model will be trained to explain the relationship between the energy consumption and the OHS profiles of the room (e.g., the indoor temperature, outdoor temperature, and past and forecast temperature), while time series models will be used to predict the future temperature. For the regression model, the overall energy cost of a room for a whole day is set to be the target variable, and this will be learnt and optimised based on the OHS profiles, which are used as independent variables. The prediction of the output values will also allow for forecasting to occur. Future cost prediction will be made by correlating past costs with the predicted future energy usage to find future costs.

The general form of the linear regression model, given a data set $\left\{y_{i}, x_{1}, \ldots, x_{i}\right\}_{i=1}^{n}$ for $n$ independent variables, can be written as:

$$
y_{i}=\beta_{0}+\beta_{1} \cdot x_{1}+\ldots+\beta_{i} \cdot x_{i}+\ldots+\beta_{n} \cdot x_{n}+\varepsilon
$$

where $x_{1}, \ldots, x_{n}$ are the independent variables, $\beta_{0}, \ldots, \beta_{n}$ are the regression coefficients, $y_{i}$ is the estimated outcome variable, and $\varepsilon$ is the error capturing the other factors that influence the $y$ other than those considered within $\left\{x_{i}\right\}_{i=1}^{n}$.

In this paper, the variables were constructed as in Figure 4 , in which we consider the following independent variables as input, including the maximum/minimum outdoor/indoor temperature of the past five days $\left(\operatorname{OTmax}_{i}, \operatorname{OTmin}_{i}, \operatorname{ITmax}_{i}, \operatorname{ITmin}_{i}, i \in\{i=-5,-4, \ldots,-1\}\right)$, the outdoor temperature in the past five hours $\left(O T_{j}, j \in\{i=-5,-4, \ldots,-1\}\right)$, the preferred room temperature of the user in the past five hours $\left(P T_{j}, j \in\{i=-5,-4, \ldots,-1\}\right)$, and the current indoor temperature $\left(I T_{c r t}\right)$, outdoor temperature $\left(O T_{c r t}\right)$, and current preferred room temperature $\left(P T_{c r t}\right)$.

The time series models were used to predict the indoor temperature $\left(I T_{p d t}\right)$, outdoor temperature $\left(O T_{p d t}\right)$, and preferred room temperature $\left(P T_{p d t}\right)$ based on the corresponding 
variables in the past days and hours. Lastly, all the collected and predicted data were fed into a regression model as independent variables, and the dependent variable was the overall energy cost of a room for a day, including the time when the room was used and not used.

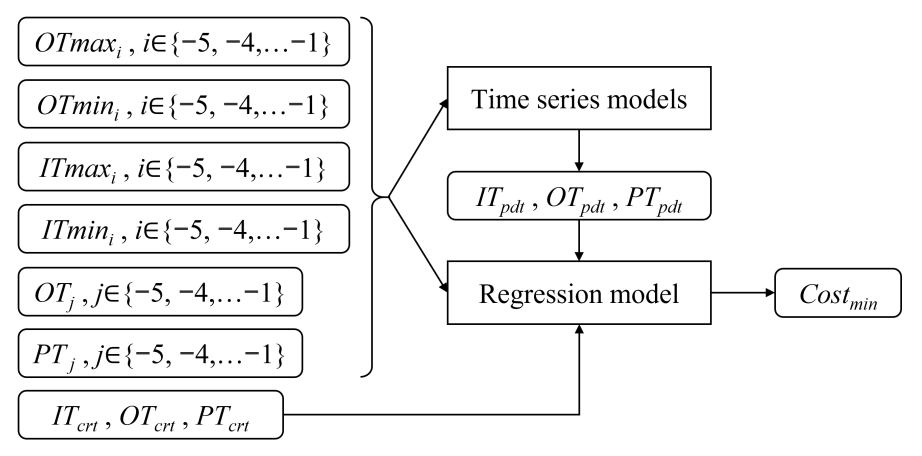

Figure 4. Machine learning models. The inputs and outputs of the models are in the rounded rectangles.

The cost prediction needs to give multiple estimates to account for a best to worst case scenario depending on how much variation is in the previous values that were used in the cost calculation. The aim is to set the $I T_{c r t}$ at a dynamic temperature to achieve the minimised Cost. In order to search for the optimised thermostat setback temperature for each room, we used the historical outdoor temperature and initiated the $I T_{c r t}$ and $P T_{c r t}$ using random numbers within a reasonable range to start up the training progress.

As for the $I T_{c r t}$, it was set within a range from 17 to $33^{\circ} \mathrm{C}$, and the $P T_{c r t}$ was set within 22 to $28^{\circ} \mathrm{C}$, because the historical data showed that most users set the air conditioner at 22 to $28^{\circ} \mathrm{C}$ after coming back to the room, and 5 extra degrees to both ends were extended for the air conditioner to handle extreme climates in the winter and summer. $N$, the number of $I T_{c r t}$, and $M$, the number of $P T_{c r t}$, were randomly generated for initial training, and the room occupation hours were also random numbers reflecting that the users could come back to the room at any time of day. The room needed to reach the $P T_{c r t}$ within $20 \mathrm{~min}$ after the $P T_{c r t}$ was set. Algorithm 1 summarises the details to search for the optimised energy cost.

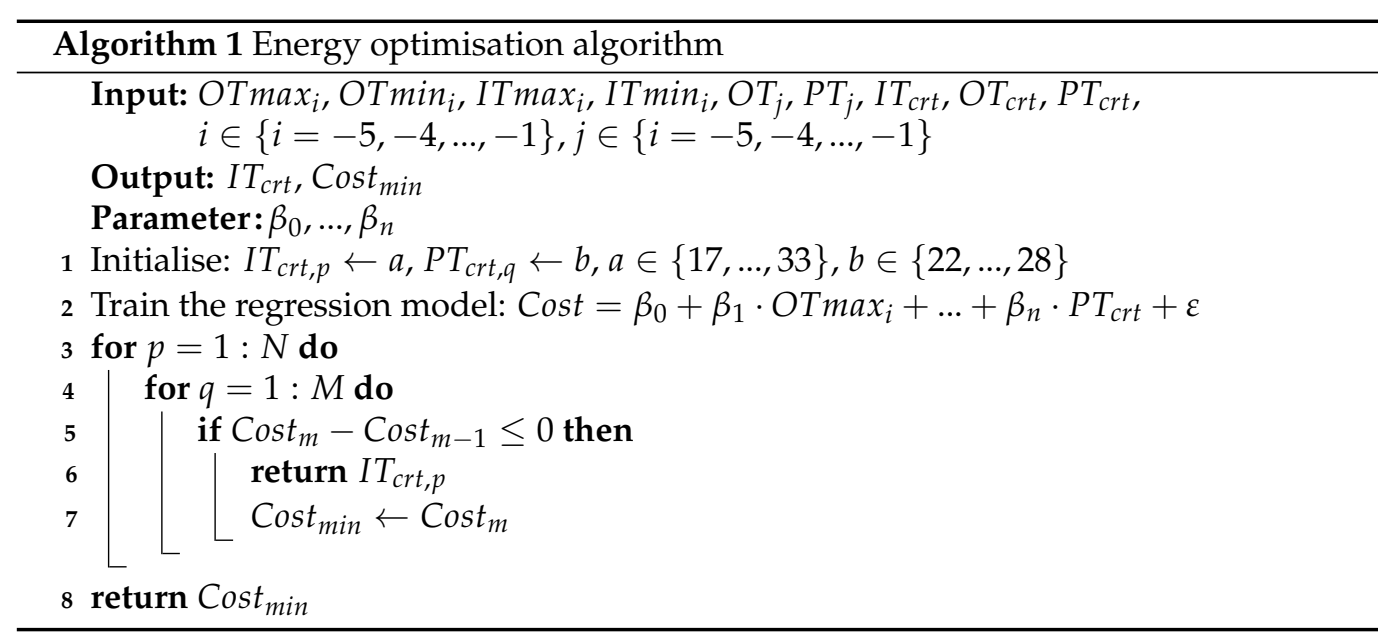

\subsection{Cyber Security and COVID-19 Response}

In the AECPE, security is handled by a set of firewalls on top of the systems being accessible only via a VPN. For the moment, whilst the system is still in development and only deployed very locally within one accommodation site, this security has proven to be sufficient; however, it will need to be improved with a proper cyber threat response system when it is ready to deploy to more locations. The security will mostly focus on the protection of the end sensors/actuators as was outlined in the replay attack section. 
As for the COVID-19 response, the movement of the people around the site can be tracked using their ID cards when they tap them to gain access to their rooms and to other common facilities. In this way, if a case of COVID-19 is detected, this person's close contacts and the areas he or she has been in during any requested time period can be quickly queried via the system. The system can also be customised to include temperature checks to scan on the arrival of the workers and other random checks throughout their time on site.

A body temperature threshold $\left(37.8^{\circ} \mathrm{C}\right)$ will be set up to trigger the system to start recording a person's temperature, and an alarm will be sent to this person and campsite manager if a body temperature above $38.28{ }^{\circ} \mathrm{C}$ [32] is detected; meanwhile, the user profile (movement and occupancy) of this person will be flagged in the system and ready to conduct contact tracing for a fast COVID-19 response.

\section{Prototype Validation and Field Testing}

\subsection{AECPE Laboratory Prototype Setup}

A prototype system was set up and tested in our lab environment in Australia. Four different devices were connected onto the system at the one time as demonstrated in Figure 5. The system could then measure how much electricity was being used and also provided remote control of the devices. On a small scale, it was shown that the system operated as was expected. Further research will show if this control functionality can be used within the system when automated commands are given instead of user commands. It will also be interesting to determine to what extend feedback loops can be used and when a user needs to step in to give new commands.

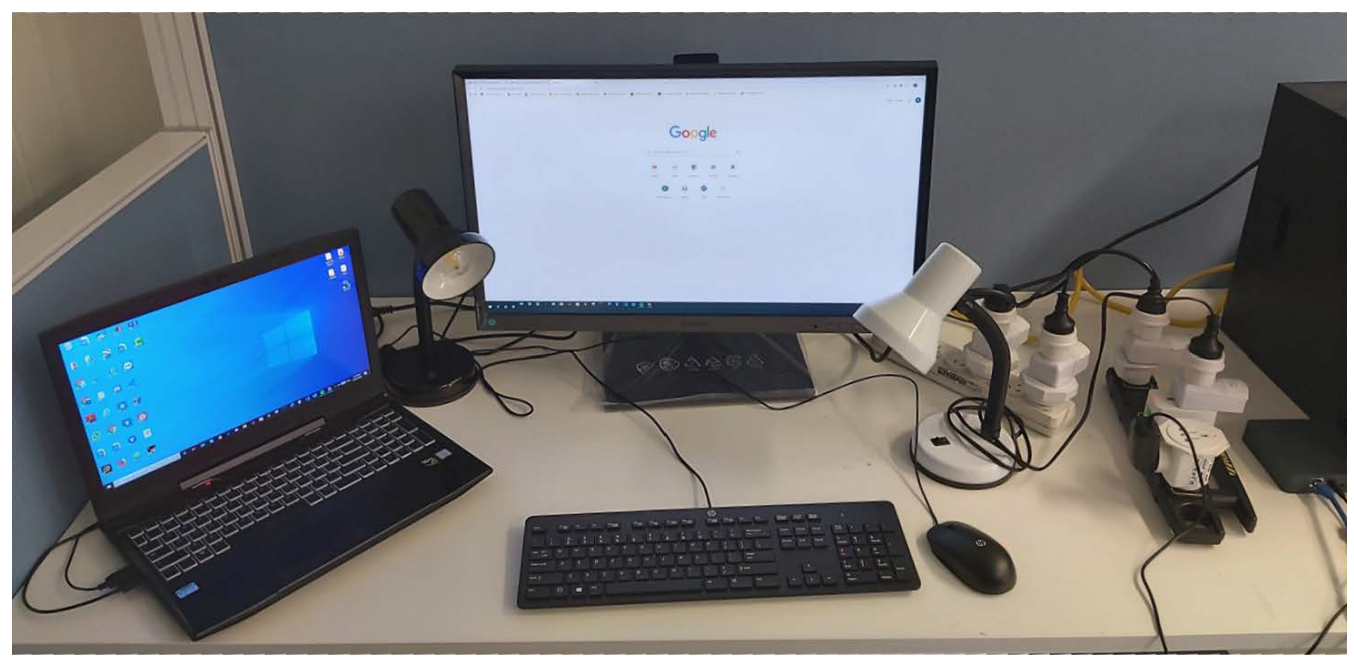

Figure 5. The lab testing setup where four plugs and a gateway (on the right) are connected to a laptop, a screen, and two desk lamps. Their energy usage data was collected and remote control was established over those devices.

\subsection{AECPE Field Testing}

We further conducted validation of the AECPE prototype in field testing in a large mining campsite provider and manager enterprise in West Australia. Specifically, the features of continuously optimising energy usage and OHS awareness were tested in one of the campsites provided and managed by the enterprise. Since this campsite is composed of a considerably large size of more than 1000 units meaning more than 3000 plus devices were connected, we also investigated the communication amongst sensors and smart meters, as well as how much the mesh network could be stretched.

In the field testing, the AECPE mainly monitored workers in and out of mining camp/room for the optimal use of electricity, and this was trialled in over 600 rooms in the mining campsite. The preliminary results demonstrated that the energy costs could be 
reduced by $50 \%$ within each camp as the system could manage the energy usage at each individual occupancy zone rather than control the energy usage in parts of the complex or part of the site/camp/building/floor/area to reduce energy waste.

In the current situation, all the appliances operated all the time regardless of the occupancy, which led to a significant amount of energy waste. Each kilowatt per hour of energy costs $\$ 0.30$ and produces approximate 1 kilo of carbon dioxide every day. If a typical mining campsite has 1000 rooms/units, with a savings of $\$ 1$ per room/per day, the AECPE could save at least $\$ 365,000$ p.a./per camp. These savings happen through only running the air-conditioning as much as is needed. Testing has shown that it tends to be more efficient to maintain the room at a temperature ranging from $24^{\circ} \mathrm{C}$ to $30^{\circ} \mathrm{C}$.

This is due to the inside room temperature getting so high (possibly up to $50{ }^{\circ} \mathrm{C}$ ) that it takes more energy to cool the room down to $19^{\circ} \mathrm{C}$ again from that temperature instead of the amount of energy that required to maintain $28{ }^{\circ} \mathrm{C}$ and then cool down to $19{ }^{\circ} \mathrm{C}$. For early testing, this was done by creating a few preset temperature states and preset scheduling. The AI algorithm has to make real time decision with the incoming temperature and roster data to ensure that the room is at the required temperature when the worker returns and also for what temperature to hold the room at while the worker is away.

Australia currently runs over 350 such mine sites [33], which all have accommodation camps associated with them. As of 2020, there were 261,900 miners working in the mining industry in Australia [34]. Applying the AECPE will result in millions of dollars saved, reduced energy consumption, and minimised carbon emissions. These figures would result in a reduction of approximately 1200 tonnes of carbon dioxide emissions p.a./per camp. This calculation is only considering the habitable facility and the mining camp and not the corporate buildings, floors, or areas.

Figures 6 and 7 demonstrate a session of our field testing where the power consumption of four different air-conditioning units in four rooms was tested and recorded over April and October reflecting two different seasons in the remote areas of Australia. The solid line refers to the unit with the proposed AECPE installed for its temperature and energy usage control, and the dashed lines denote those without instructional operation or relying on a rudimentary energy saving algorithm. Overall, AECPE had a comparatively lower energy usage than the other air-conditioning units. Specifically, it used $40 \%$ of AC1's power consumption, $47 \%$ of AC2's power consumption, and 46\% of AC3's power consumption on average throughout the month.

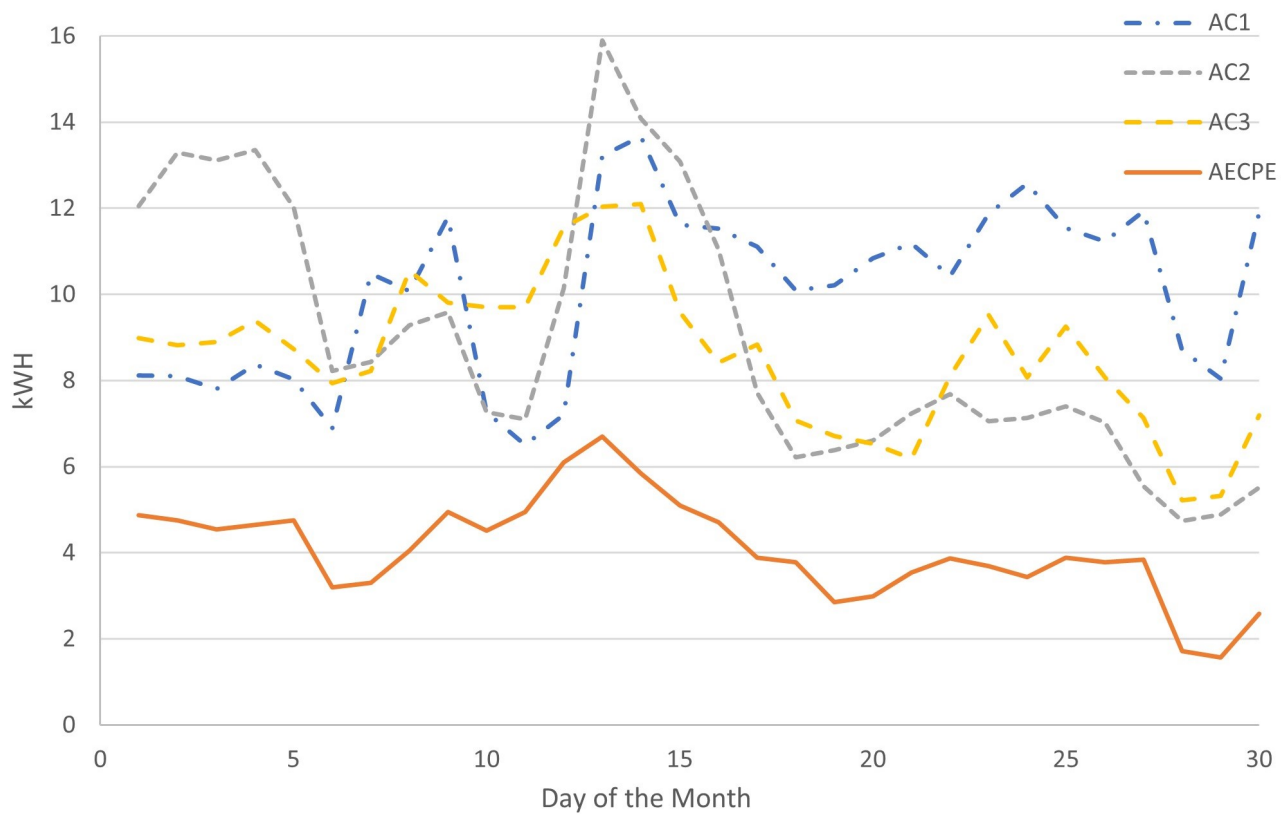

Figure 6. April results from the field test. 


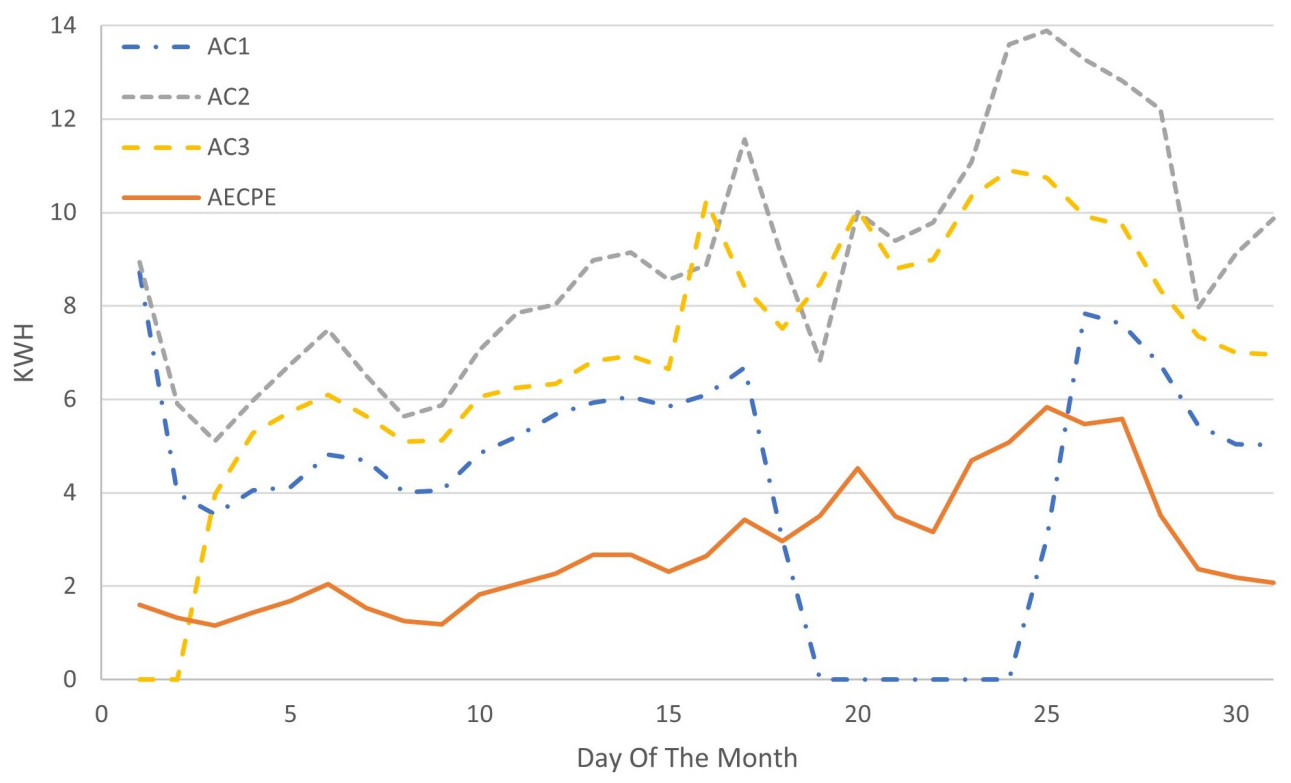

Figure 7. October results from the field test.

\section{Conclusions}

We developed an AI-Empowered Cyber-Physical Ecosystem (AECPE) that is capable of monitoring energy consumption in real time and maintaining OHS parameters. This system aims to assist in achieving energy efficiency and better OHS outcomes for workers and facilities in remote regions. The combined energy and OHS technology will help remote operations in not only energy waste reduction but also a better understanding of energy usage and OHS needs. A CPS- and AI-based platform was designed to provide integrated analytics enabling the efficient management of facilities, including energy consumption, improved OHS, emission reductions, efficient resource utilisation, and efficient facility maintenance, along with timely decision support between differing autonomous CPS devices, including smart metering systems.

The AECPE is based on the integration of smart metering and machine-learning-based optimisation with OHS. Bringing these elements together is important as it will allow for the system to run in its most efficient state. This combination will allow this system to provide a new way of controlling and overseeing the OHS of a site over a mobile phone. Using a new generation of smart meters, it will allow measurements to be taken at a faster speed than any other smart meters (as fast as one every $20 \mathrm{~ms}$ ) with an accuracy of $99 \%$.

Measurements taken this frequently will require a greater amount of storage if the internet connection is to ever drop out at a remote site. The gateway will provide this storage, as it can store the data from multiple days until it becomes reconnected to the internet. This would allow for no loss of data to occur to ensure accurate analysis and future predictions. The system is also capable of responding to the COVID-19 pandemic in terms of user monitoring and contact tracing, which is aligned with the government policies regarding the OHS during this pandemic period.

For future work, we will work on developing machine learning and predictive analytical algorithms for pattern recognition and integrated analytics for the two interlinking areas, namely energy consumption and OHS, to help optimise energy usage and eliminate waste while maintaining health and well-being standards. In addition, we will also provide energy and OHS training programs for senior managers in the remote mining organisations and assist them with low-cost smart mining camp solutions based on our collected data and analysis results. The cyber security system will also need to be updated as the work goes along to keep up with potential threats. 
Author Contributions: Conceptualization: P.K., S.W. and E.C.; Methodology: P.K., Y.Z. and E.C.; Software: P.K.; Validation: P.K. and S.W.; Investigation: P.K. and Y.Z.; Resources: P.K., S.W. and E.C.; Writing—original draft preparation: P.K.; Writing—review and editing: P.K., Y.Z. and E.C.; Supervision: Y.Z. and E.C. All authors have read and agreed to the published version of the manuscript.

Funding: This research received no external funding.

Conflicts of Interest: The authors declare no conflict of interest.

\section{References}

1. Stellman, J.M. Encyclopaedia of Occupational Health and Safety; International Labour Organization: Geneva, Switzerland, 1998; Volume 1.

2. Chang, E.; Gottwalt, F.; Zhang, Y. Cyber situational awareness for CPS, 5G and IoT. In Frontiers in Electronic Technologies; Springer: Singapore, 2017; pp. 147-161.

3. Lee, J.; Bagheri, B.; Kao, H.A. A cyber-physical systems architecture for industry 4.0-based manufacturing systems. Manuf. Lett. 2015, 3, 18-23. [CrossRef]

4. Horcas, J.M.; Pinto, M.; Fuentes, L. Context-aware energy-efficient applications for cyber-physical systems. Ad Hoc Netw. 2019, 82, 15-30. [CrossRef]

5. Bartocci, E.; Deshmukh, J.; Donzé, A.; Fainekos, G.; Maler, O.; Ničković, D.; Sankaranarayanan, S. Specification-based monitoring of cyber-physical systems: A survey on theory, tools and applications. In Lectures on Runtime Verification; Springer: Cham, Switzerland, 2018; pp. 135-175.

6. Dey, N.; Ashour, A.S.; Shi, F.; Fong, S.J.; Tavares, J.M.R. Medical cyber-physical systems: A survey. J. Med. Syst. 2018, $42,74$. [CrossRef] [PubMed]

7. Wang, J.; Abid, H.; Lee, S.; Shu, L.; Xia, F. A secured health care application architecture for cyber-physical systems. arXiv 2011, arXiv:1201.0213.

8. Banerjee, A.; Gupta, S.K.; Fainekos, G.; Varsamopoulos, G. Towards modeling and analysis of cyber-physical medical systems. In Proceedings of the 4th International Symposium on Applied Sciences in Biomedical and Communication Technologies, Barcelona, Spain, 26-29 October 2011; pp. 1-5.

9. Wahid, F.; Kim, D.H. An Efficient Approach for Energy Consumption Optimization and Management in Residential Building Using Artificial Bee Colony and Fuzzy Logic. Math. Probl. Eng. 2016, 2016, 9104735. [CrossRef]

10. Attia, A.H.; Rezeka, S.F.; Saleh, A.M. Fuzzy logic control of air-conditioning system in residential buildings. Alex. Eng. J. 2015, 54, 395-403. [CrossRef]

11. Patanaik, A. Fuzzy Logic Control of Air Conditioners; Indian Institute of Technology: Kharagpur, India, 2008.

12. Kale, S.S.; Patil, P.S. Data mining technology with fuzzy logic, neural networks and machine learning for agriculture. In Data Management, Analytics and Innovation; Springer: Singapore, 2019; pp. 79-87.

13. Past Weather in Karratha, Western Australia, Australia. Available online: https://www.timeanddate.com/weather/australia/ karratha/historic (accessed on 4 June 2021).

14. Hasbullah, H.; Soomro, I.A. Denial of service (DOS) attack and its possible solutions in VANET. Int. J. Electron. Commun. Eng. 2010, 4, 813-817.

15. Bicakci, K.; Tavli, B. Denial-of-Service attacks and countermeasures in IEEE 802.11 wireless networks. Comput. Stand. Interfaces 2009, 31, 931-941. [CrossRef]

16. Krotofil, M.; Cárdenas, A.A.; Manning, B.; Larsen, J. CPS: Driving Cyber-Physical Systems to Unsafe Operating Conditions by Timing DoS Attacks on Sensor Signals. In Proceedings of the 30th Annual Computer Security Applications Conference (ACSAC '14); Association for Computing Machinery: New York, NY, USA, 2014; pp. 146-155. [CrossRef]

17. Nagarsheth, P.; Khoury, E.; Patil, K.; Garland, M. Replay Attack Detection Using DNN for Channel Discrimination. In Proceedings of the Interspeech 2017-18th Annual Conference of the International Speech Communication Association, Stockholm, Sweden, 18-24 August 2017; pp. 97-101.

18. Hosseinzadeh, M.; Sinopoli, B.; Garone, E. Feasibility and Detection of Replay Attack in Networked Constrained Cyber-Physical Systems. In Proceedings of the 2019 57th Annual Allerton Conference on Communication, Control, and Computing (Allerton), Monticello, IL, USA, 24-27 September 2019; pp. 712-717. [CrossRef]

19. Liu, Y.; Ning, P.; Reiter, M.K. False Data Injection Attacks against State Estimation in Electric Power Grids. ACM Trans. Inf. Syst. Secur. 2011, 14. [CrossRef]

20. Ahmed, M.; Pathan, A.S.K. False data injection attack (FDIA): An overview and new metrics for fair evaluation of its countermeasure. Complex Adapt. Syst. Model. 2020, 8. [CrossRef]

21. Mpelogianni, V.; Groumpos, P.; Tsipianitis, D.; Papagiannaki, A.; Gionas, J. Proactive Building Energy Management based on Fuzzy Logic and Expert Intelligence. Inform. Intell. Syst. Appl. 2020, 1, 56-58. [CrossRef]

22. Paxton. Access Control. Available online: https://www.paxton-access.com/solutions/access-control/ (accessed on 8 April 2021).

23. KBE Building Corporation. Available online: https://kbebuilding.com/portfolio/ (accessed on 8 April 2021).

24. Zhang, Y.; Guo, Z.; Lv, J.; Liu, Y. A Framework for Smart Production-Logistics Systems Based on CPS and Industrial IoT. IEEE Trans. Ind. Inform. 2018, 14, 4019-4032. [CrossRef] 
25. Törngren, M.; Asplund, F.; Bensalem, S.; McDermid, J.; Passerone, R.; Pfeifer, H.; Sangiovanni-Vincentelli, A.; Schätz, B. Characterization, analysis, and recommendations for exploiting the opportunities of cyber-physical systems. In Cyber-Physical Systems; Elsevier: Amsterdam, The Netherlands, 2017; pp. 3-14.

26. Hernández-Muñoz, J.M.; Vercher, J.B.; Muñoz, L.; Galache, J.A.; Presser, M.; Gómez, L.A.H.; Pettersson, J. Smart cities at the forefront of the future internet. In Future Internet Assembly; Springer: Berlin/Heidelberg, Germany, 2011 ; pp. 447-462.

27. Bouchama, A.; Knochel, J.P. Heat Stroke. N. Engl. J. Med. 2002, 346, 1978-1988. [CrossRef] [PubMed]

28. Nguyen, J.L.; Schwartz, J.; Dockery, D.W. The relationship between indoor and outdoor temperature, apparent temperature, relative humidity, and absolute humidity. Indoor Air 2014, 24, 103-112. [CrossRef] [PubMed]

29. Karratha Climate. Available online: https://en.climate-data.org/oceania/australia/western-australia/karratha-11105/ (accessed on 29 May 2021).

30. Zigbee Pro Best Practises. Available online: https://www.control4.com/docs/product/zigbee/best-practices/english/revision/ A/zigbee-best-practices-rev-a.pdf (accessed on 29 May 2021).

31. Binder, J.J. On the use of the multivariate regression model in event studies. J. Account. Res. 1985, 23, 370-383. [CrossRef]

32. What Temperature Is Considered a Fever? Available online: https://www.singlecare.com/blog/fever-temperature/ (accessed on 14 June 2021).

33. Australian Mineral Facts. Available online: https://www.ga.gov.au/education/classroom-resources/minerals-energy/ australian-mineral-facts\#: :text=Source \%20Geoscience \%20Australia.,from\%20over\%20350\%20operating $\% 20 \mathrm{mines}$ (accessed on 28 May 2021).

34. In Numbers: How Mining Came to be Australia's Most Profitable Sector. Available online: https:/ / www.mining-technology. com/features/in-numbers-how-mining-came-to-be-australias-most-profitable-sector/ (accessed on 28 May 2021). 